in vivo $33: 1203-1208$ (2019)

doi:10.21873/invivo.11591

\title{
Effects of Far-infrared Ray on Temozolomide-treated Glioma in Rats
}

\author{
JIN-CHERNG CHEN ${ }^{1,2}$ and JUEN-HAUR HWANG ${ }^{2,3,4}$ \\ ${ }^{1}$ Department of Neurosurgery, Dalin Tzu Chi Hospital, \\ Buddhist Tzu Chi Medical Foundation, Chiayi, Taiwan, R.O.C.; \\ ${ }^{2}$ School of Medicine, Tzu Chi University, Hualien, Taiwan, R.O.C.; \\ ${ }^{3}$ Department of Otolaryngology-Head and Neck Surgery, \\ Dalin Tzu Chi Hospital, Buddhist Tzu Chi Medical Foundation, Chiayi, Taiwan, R.O.C.; \\ ${ }^{4}$ Department of Medical Research, China Medical University Hospital, \\ China Medical University, Taichung, Taiwan, R.O.C.
}

\begin{abstract}
Background/Aim: Malignant glioma is a rapidly progressive primary brain cancer. The aim of the study was to investigate the effect of far-infrared ray (FIR) on temozolomide (TMZ)-treated glioma in rats. Materials and Methods: Male, 8-week old, Fischer 344 inbred rats with glioma were randomly divided into three study groups (20 rats in each group). The control group received saline only once daily for 5 days. The TMZ group received TMZ (30 $m g / k g$ ) once daily for 5 days. The TMZ plus FIR group received TMZ (30 mg/kg) once daily for 5 days and infrared-c irradiation of 40 min twice daily for 4 weeks. The relative tumor fold and the expression of hypoxia-induced factor-1 $\alpha(H I F-1 \alpha)$ and vascular endothelial growth factor (VEGF) were compared using one-way ANOVA at the end of study. Results: The relative tumor fold of the TMZ+FIR group was significantly higher compared to the control group, and was borderline higher compared to the TMZ group at Day 7. The relative tumor fold of TMZ+FIR group was significantly higher compared to the control group and the TMZ group at Days 14, 21 and 28. HIF-1 $\alpha$ expression of TMZ+FIR group was borderline higher compared to the control group at Day 28. The VEGF expression of $T M Z+F I R$ group was significantly higher compared to the
\end{abstract}

This article is freely accessible online.

Correspondence to: Juen-Haur Hwang, Department of Otolaryngology-Head and Neck Surgery, Dalin Tzu Chi Hospital, Buddhist Tzu Chi Medical Foundation, No. 2 Minsheng Road, Dalin, Chiayi, 62247, Taiwan, R.O.C. Tel: +886 52648000, Fax: +886 52648006, e-mail: g120796@tzuchi.com.tw

Key Words: Far-infrared ray, glioma, hypoxia-induced factor-1 $\alpha$, vascular endothelial growth factor, temozolomide. control group and the TMZ group at Day 28. Conclusion: FIR might increase the growth of glioma under TMZ treatment in rats possibly via increasing VEGF expression, but not HIF-1 $\alpha$ expression.

Malignant glioma is the most common primary brain cancer in adults. Despite many efforts, treatment of glioblastoma multiforme (GBM) remains one of the most challenging tasks. Poor cancer location, opportunistic deletion of tumor suppressor genes, amplification and/or mutational hyper-activation of receptor tyrosine kinase receptors, defects in the apoptosis signaling machinery, rapid growth rate, and poor response to current treatment modalities, might account for the poor prognosis of malignant glioma $(1,2)$.

The status of oxygenation in the tumor microenvironment may also affect the prognosis of cancer patients. Hypoxia is a major regulator of tumor development and aggressiveness. The two principal mediators of hypoxia response, hypoxiainduced factor-1 and -2 (HIF-1 and HIF-2), are known to have different responses to hypoxia. Stromal HIF-1 and HIF-2 have been reported to have opposing roles in breast cancer progression (3), while intralesional hypoxia near areas of necrosis with high levels of HIF-1 has been associated with poor prognosis in breast cancer (3). The most hypoxic and immature GBM cells are resistant to temozolomide (TMZ) due to high expression of HIF- $1 \alpha$ and O6-methylguanine-DNA-methyltransferase (MGMT) (4). A previous study has shown that intermittent hypoxia increases tumor growth in a mouse model of sleep apnea, and circulating vascular endothelial growth factor (VEGF) appears to be a crucial mediator of tumor growth (5). Alveolar hypoxia promotes murine lung tumor growth through a VEGFR-2/epidermal growth factor receptor (EGFR)-dependent mechanism (6). Similarly, angiogenesis 
is a significant feature of GBM (7). Exosomes derived from GBM cells grown at hypoxic compared to normoxic conditions are potent inducers of angiogenesis both ex vivo and in vitro through a phenotypic modulation of endothelial cells (8). Additionally, EGF activation of EGF receptor expressed on glioma cells leads to enhanced secretion of VEGF by glioma cells (9). VEGF has, thus, become a new target in the treatment of $\operatorname{GBM}(7,10)$.

Taken together, it is reasonable to expect that interventions affecting glioma oxygenation status and/or VEGF expression might affect the prognosis of glioma in some aspects. Infrared-C (IR-C) radiation (usually referred to as far-infrared ray, FIR) is non-ionizing radiation with wavelengths of 3-1,000 $\mu \mathrm{m}$ in the infrared band as defined by the International Commission on Illumination (11). In the IR-C band, the FIR of 4-14 $\mu \mathrm{m}$ is also called "growth rays" due to its several advantageous effects in several organisms $(12,13)$. IR-C radiation can promote blood circulation, reduce oxidative stress and relieve pain, either by thermal or through non-thermal regulations in animals or humans $(14,15)$. Also, sleep disorders, high sympathetic tone and hypertension can be released by IRC treatment (16).

Despite all the benefits, heat and FIR are not totally harmless on living tissues. FIR might be involved in both photo-aging and photo-carcinogenesis (17). With some contradictory biological effects, the impact of FIR on glioma still remains unknown. In this study, therefore, we aimed to evaluate the effects of applying FIR in TMZ-treated glioma in rats.

\section{Materials and Methods}

Cell line and cell culture. RT2 was kindly provided by National Taiwan University Hospital (Taipei, Taiwan, ROC). Cells were maintained in Dulbecco's Modified Eagle Medium (Gibco, Berlin, Germany) that was supplemented with L-glutamine, streptomycin, penicillin (Gibco, Berlin, Germany) and $10 \%$ fetal calf serum (Gibco, Berlin, Germany). All cells were cultured at $37^{\circ} \mathrm{C}$ in a $5 \%$ $\mathrm{CO}_{2}$ incubator.

Animal care and study. The study protocol was approved by the Institutional Animal Care and Use Committee of the Dalin Tzu Chi Hospital, Buddhist Tzu Chi Medical Foundation, Chiayi, Taiwan, R.O.C. (1031003-2).

Male, 8-week old, Fischer 344 inbred rat were purchased from the National Laboratory Animal Center (Taipei, Taiwan, ROC). Food and water were provided ad libitum to the animals in standard cages. The rats were maintained in a daily cycle of 12-h period of light and darkness. Animals were monitored for morbidity or signs of distress throughout the study. Humane endpoints for these studies were based on weight loss (>10-15\%) and clinical observations for behavior (decreased activity, hunched posture, shivering, labored breathing, moribund). We did not observe any adverse events during our studies, including signs of illness or mortality prior to the experimental endpoint.
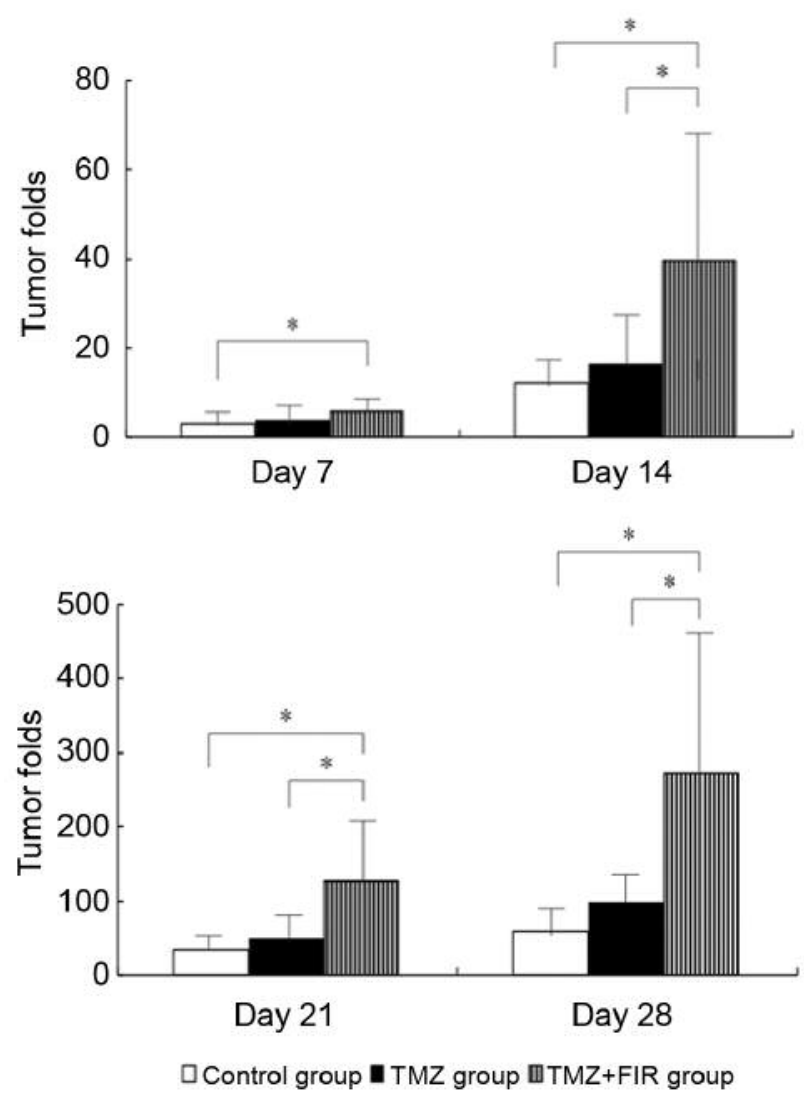

Figure 1. Relative tumor folds each week in the three groups: i) control, ii) $T M Z$ groupd, and iii) $T M Z+F I R$. The relative tumor fold of $T M Z+F I R$ group was significantly higher compared to the control group, and was borderline higher compared to the TMZ group on Day 7 ( $p=0.013$ and 0.086 , respectively). The relative tumor fold of $T M Z+F I R$ group was significantly higher compared to the control group and the TMZ group on Day 14 ( $p<0.001$ and 0.001, respectively), on Day 21 ( $p<0.001$ for both comparisons), and Day 28 ( $p<0.001$ for both comparisons). TMZ: Temozolomide; FIR: far-infrared ray.

Development of subcutaneous tumors. RT 2 cells $\left(1 \times 10^{7}\right.$ cells in 500 $\mu \mathrm{l}$ per injection site) were suspended in phosphate-buffered saline (PBS). Rat were anesthetized by continuous flow of 3\% isofluorane (2.5\% LPM oxygen), and RT2 glioma cells were implanted into the subcutaneous space of right flank in Fischer 344 rats. Animals were observed for 45 to $60 \mathrm{~min}$ following tumor implantation, until they fully recovered.

Tumor growth was monitored using a digital caliper (H\&H 161506, Taiwan, ROC) every week. Tumor volume was estimated according to the following formula: Volume $=0.5 *(\mathrm{~d} 1 * \mathrm{~d} 2 * \mathrm{~d} 3)$, in which $\mathrm{d} 1, \mathrm{~d} 2$ and $\mathrm{d} 3$ are the three dimensions of the tumor, respectively (18).

Grouping and treatments. Two weeks later, rats with tumor volume greater than $62.5 \mathrm{~mm}^{3}$ were randomly and equally divided into three study groups (20 rats in each group). Rats were treated by oral instillation of saline or TMZ, and/or IR-C irradiation (Far 


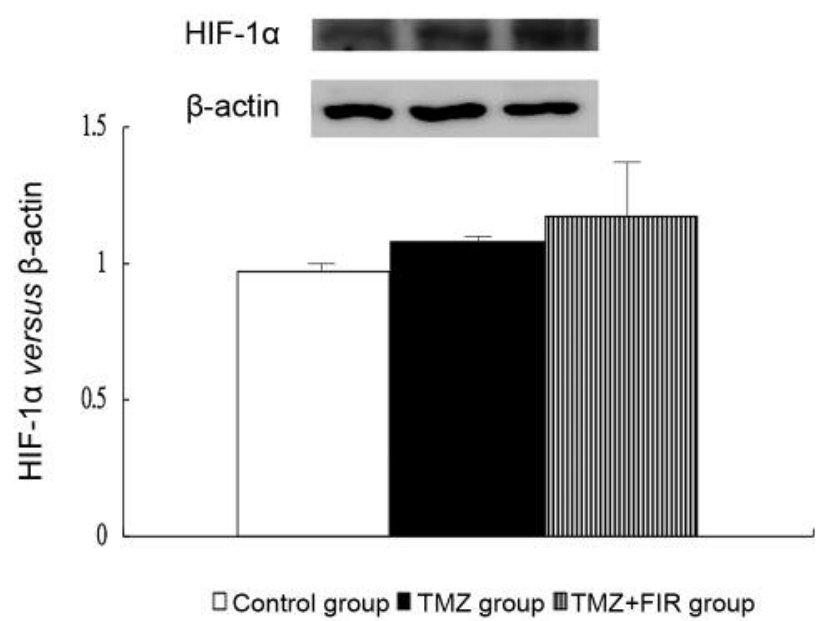

Figure 2. Results of western blotting of HIF-1 $\alpha$ in the three groups: $i)$ control, ii) TMZ groupd, and iii) TMZ + FIR at the end of the study. The HIF-1 $\alpha$ expression of TMZ+FIR group was borderline higher compared to the control group on Day 28. The HIF-1 $\alpha$ expression of $T M Z$ group was not significantly higher compared to the control group on Day 28. TMZ: Temozolomide; FIR: far-infrared ray.

Infrared Physiatric Unit TY-101F, WS FAR IR MEDICAL TECHNOLOGY CO., LTD). The control group received only saline once daily for 5 days. The TMZ group received TMZ (30 $\mathrm{mg} / \mathrm{kg}$ ) once daily for 5 days. The TMZ plus FIR group received TMZ (30 mg/kg) once daily for 5 days and IR-C irradiation of 40 min twice daily for 4 weeks.

The relative tumor fold was calculated by the tumor volume measured each week compared to that at the start of this study (18).

Western blotting analysis. All rats were sacrificed by inhalation of $5 \%$ isoflurane followed by decapitation at the end of the study (4 weeks after start of this study and 6 weeks following the injection of RT2 cells). Tumor masses were collected and snap-frozen in liquid nitrogen and were stored at $-80^{\circ} \mathrm{C}$ until analysis.

Sections of the tumor masses were washed in PBS, and broken into small tumor pieces using scissors in $0.2 \mathrm{ml}$ T-PER tissue protein extraction reagent $\left(\mathrm{T}-\mathrm{PER}^{\mathrm{TM}}\right.$ Tissue Protein Extraction Reagent, Thermo Fisher Scientific Inc., USA), according to the manufacturer's instructions.

The protein concentration was determined using the BCA protein quantitative method (Pierce ${ }^{\mathrm{TM}}$ BCA Protein Assay Kit, Thermo Fisher Scientific Inc., USA). After SDS-PAGE (sodium dodecyl sulfate-polyacrylamide gel electrophoresis) protein electrophoresis, proteins were transferred to a PVDF (polyvinylidene fluoride) membrane. Then, $1 \times$ Tris-buffered saline with $0.1 \%$ Tween-20 (TBS-T) containing 5\% skim milk was added to block the membrane for 1 hour. Membranes were washed three times in TBS$\mathrm{T}$ for ten min each. Subsequently, primary antibodies of HIF-1 $\alpha$ (1:1,000, Abcam plc, UK), VEGF (1:5,000, Merck Millipore, USA) and $\beta$-actin $(1: 5,000$, Sigma-Aldrich, USA), were diluted in TBS-T containing $5 \%$ non-fat dry milk, were added to the membrane and were incubated overnight at $4^{\circ} \mathrm{C}$. Membranes were washed three

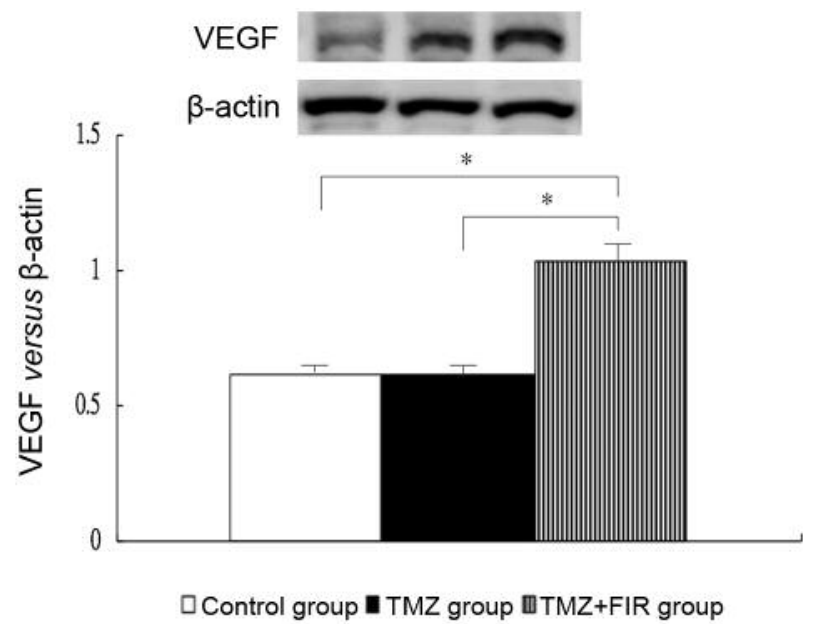

Figure 3. Results of western blotting of VEGF in the three groups: i) control, ii) TMZ groupd, and iii) TMZ+FIR at the end of the study. The VEGF expression of TMZ+FIR group was significantly higher compared to the control group and the TMZ group on Day 28 ( $p=0.001$ for both comparisons). The VEGF expression of TMZ group was not significantly higher compared to the control group on Day 28. TMZ: Temozolomide; FIR: far-infrared ray.

times in TBS-T. Subsequently, horseradish peroxidase (HPR)conjugated secondary antibodies in TBS-T with 5\% skim milk were added to the membrane and were incubated for 1 hour. After incubation, Membranes were washed with TBS-T. The film was finally developed using enhanced chemiluminescence (ECL) (WesternBright ECL HRP substrate, Advansta Inc, USA). Membranes were visualized and analysed with an Odyssey ${ }^{\circledR} \mathrm{Fc}$ Imaging System and Image Studio ${ }^{\mathrm{TM}}$ Software and organization and with Empiria Studio ${ }^{\mathrm{TM}}$ Software for analysis (LI-COR Biosciences, USA). The internal control was $\beta$-actin.

Statistical analysis. The relative tumor folds, and protein expression of HIF- $1 \alpha$ and VEGF relative to that of $\beta$-actin, were compared between the three groups using one-way analysis of variance (ANOVA) with a post-hoc Bonferroni correction. All analyses were performed using the commercial software Stata (StataCorp LLC, College Station, TX, USA), and $p$-Values $<0.05$ were considered statistically significant.

\section{Results}

The general condition and body weight in all three groups were similar before and after the experiment. No animal in the three groups expired or experiences adverse effects during any of the experimental procedures (20 rats survived in each group).

Figure 1 shows the relative tumor folds during each week in the three groups. The relative tumor fold of TMZ+FIR group was significantly higher compared to the control group, and borderline higher compared to the TMZ group 
at Day 7 (one-way ANOVA, $p=0.0117$ and post-hoc Bonferroni correction, $p=0.013$ and 0.086 , respectively). The relative tumor fold of $\mathrm{TMZ}+\mathrm{FIR}$ group was significantly higher compared to the control group and the TMZ group at Day 14 (one-way ANOVA, $p<0.0001$ and post-hoc Bonferroni correction, $p<0.001$ and 0.001 , respectively), at Day 21 (one-way ANOVA, $p<0.0001$ and post-hoc Bonferroni correction, $p<0.001$ and $<0.001$, respectively), and at Day 28 (one-way ANOVA, $p<0.0001$ and post-hoc Bonferroni correction, $p<0.001$ and $<0.001$, respectively).

Figure 2 shows the results of western blotting of HIF- $1 \alpha$ in the three groups at the end of study. The HIF-1 $\alpha$ expression in the TMZ+FIR group was borderline higher compared to the control group at Day 28 (one-way ANOVA, $p=0.0821$ and post-hoc Bonferroni correction, $p=0.096$ ), while for the TMZ group HIF-1 expression was not significantly higher compared to the control group at Day 28 (post-hoc Bonferroni correction, $p=0.474$ ).

Figure 3 shows the results of the western blotting of VEGF in the three groups at the end of study. The VEGF expression in the TMZ+FIR group was significantly higher compared to the control and the TMZ groups at Day 28 (oneway ANOVA, $p=0.0006$ and post-hoc Bonferroni correction, $p=0.001$ and $p=0.001$, respectively), however, the VEGF expression in the TMZ group was not significantly higher compared to the control group at Day 28 (post-hoc Bonferroni correction, $p=1.000)$.

\section{Discussion}

This experimental study shows that FIR might increase the growth of glioma under TMZ treatment and that this might be associated with increasing VEGF expression, but not with HIF- $1 \alpha$ expression. In other words, FIR was harmful in glioma with TMZ treatment possibly via increasing VEGF expression in the lesions.

Rapid cellular proliferation, hypervascularity, cerebral edema, and focal necrosis are key histopathologic features of GBM (9). The most hypoxic and immature GBM cells are resistant to TMZ due to the high expression of HIF- $1 \alpha$ and MGMT (4). An intratumoral hypoxic gradient drives stem cell distribution and MGMT expression in glioblastoma (19). Conversely, down-modulating the HIF-1 $\alpha /$ MGMT axis might increase GBM responsiveness to chemotherapy (4). Also, glioblastoma stem-like cells secrete the pro-angiogenic VEGF-A factor in extracellular vesicles, which carry essential information that can adapt the microenvironment to the tumor's needs, inducing tumour-associated angiogenesis (20). VEGF can increase cell motility, but it does not affect cell proliferation in glioma cell lines (10). Overexpression of VEGF isoforms have been shown to drive vascularization, oxygenation, and growth but not progression to GBM in a human model of gliomagenesis (21).
Studies on phototherapy and/or irradiation in glioma are limited and diverse. Irradiation has been shown to increase cell motility, but it does not affect cell proliferation in glioma cell lines (10). Radiation-induced VEGF can also increase cell motility of glioma in vitro (22). On the contrary, photothermal ablation therapy can significantly prolong the survival of tumor-bearing mice in an orthotopic mouse xenograft model of glioma (23). Near-infrared light can trigger release of Paclitaxel from biodegradable microspheres and enhance its antitumoral activity via a photothermal effect (24). PEGylated gold nanorods and near-infrared stimulation have been shown to trigger release of doxorubicin from thermosensitive liposomes in a mouse tumor model of human glioblastoma (25), while nanoshell-mediated photothermal therapy with a near infrared laser, has improved survival in a murine glioma model (26). Collectively, it seems that phototherapy and/or irradiation itself may be harmful for cancer control, but it could enhance the effects of chemotherapy on glioma.

Concerning FIR therapy, previous studies have shown that it could increase temperature in the body tissues, elevate motility of body fluids (27), and exert a nitric oxide (NO)related biological effect to increase skin microcirculation in rats $(27,28)$. In addition, FIR may reduce the growth of some cancer cells in vitro with a low level of heat shock protein 70 (29). However, FIR might also be involved in both photo-aging and photo-carcinogenesis (17).

In our study we found that FIR can enhance the growth of glioma when using TMZ therapy possibly via increased VEGF expression. These contradictory results might be due to different cancer types, protocols of IR-C irradiation, and/or combinations of other therapy. Even though there are groups claiming that FIR may be helpful for glioma control (30) clinicians should recommend the use of FIR for glioma treatment with caution. We suggest a large-scale clinical trial on this issue in the future.

\section{Conflicts of Interest}

The Authors declare no conflicts of interest. The funders had no role in study design, data collection and analysis, decision to publish, or preparation of the manuscript.

\section{Authors' Contributions}

JCC prepared the manuscript and coordinated the study. JCC carried out the experiments. Both JCC and JHH were involved in designing the experiments. All authors read and approved the final manuscript.

\section{Acknowledgements}

This work was supported by Grants from Dalin Tzu Chi Hospital, Buddhist Tzu Chi Medical Foundation, Chiayi, Taiwan (DTCRD104-I-02-01). 


\section{References}

1 Mrugala MM: Advances and challenges in the treatment of glioblastoma: a clinician's perspective. Discov Med 15(83): 221230, 2013. PMID: 23636139.

2 Krakstad C and Chekenya M: Survival signalling and apoptosis resistance in glioblastomas: opportunities for targeted therapeutics. Mol Cancer 9: 135, 2010. PMID: 20515495. DOI: 10.1186/1476-4598-9-135

3 Rundqvist $\mathrm{H}$ and Johnson RS: Tumour oxygenation: implications for breast cancer prognosis. J Intern Med 274(2): 105-112, 2013 PMID: 23844914. DOI: 10.1111/joim.12091

4 Persano L, Pistollato F, Rampazzo E, Della Puppa A, Abbadi S, Frasson C, Volpin F, Indraccolo S, Scienza R and Basso G: BMP2 sensitizes glioblastoma stem-like cells to Temozolomide by affecting HIF- $1 \alpha$ stability and MGMT expression. Cell Death Dis 3: e412, 2012. PMID: 23076220. DOI: 10.1038/cddis. 2012.153

5 Almendros I, Montserrat JM, Torres M, Bonsignore MR, Chimenti L, Navajas D and Farre R: Obesity and intermittent hypoxia increase tumor growth in a mouse model of sleep apnea Sleep Med 13(10): 1254-1260, 2012. PMID: 23149216. DOI: 10.1016/j.sleep.2012.08.012

6 Karoor V, Le M, Merrick D, Fagan KA, Dempsey EC and Miller YE: Alveolar hypoxia promotes murine lung tumor growth through a VEGFR-2/EGFR- dependent mechanism. Cancer Prev Res (Phila) 5(8): 1061-1071, 2012. PMID: 22700853. DOI: 10.1158/1940-6207.CAPR-12-0069-T

7 Okuda T, Tasaki T, Nakata S, Yamashita K, Yoshioka H, Izumoto S, Kato A and Fujita M: Efficacy of combination therapy with MET and VEGF inhibitors for MET-overexpressing glioblastoma. Anticancer Res 37(7): 3871-3876, 2017. PMID: 28668888. DOI: 10.21873/anticanres.11767

8 Kucharzewska P, Christianson HC, Welch JE, Svensson KJ, Fredlund E, Ringnér M, Mörgelin M, Bourseau-Guilmain E, Bengzon $\mathrm{J}$ and Belting M: Exosomes reflect the hypoxic status of glioma cells and mediate hypoxia-dependent activation of vascular cells during tumor development. Proc Natl Acad Sci USA 110(18): 7312-7317, 2013. PMID: 23589885. DOI: 10.1073/pnas.122099 8110

9 Goldman CK, Kim J, Wong WL, King V, Brock T and Gillespie GY: Epidermal growth factor stimulates vascular endothelial growth factor production by human malignant glioma cells: a model of glioblastoma multiforme pathophysiology. Mol Biol Cell 4(1): 121-133, 1993. PMID: 7680247.

10 Krcek R, Matschke V, Theis V, Adamietz IA, Bühler H and Theiss C: Vascular endothelial growth factor, irradiation, and axitinib have diverse effects on motility and proliferation of glioblastoma multiforme cells. Front Oncol 7: 182, 2017. PMID: 28879167. DOI: $10.3389 /$ fonc. 2017.00182

11 Henderson R: Wavelength considerations. Instituts für Umformund Hochleistungs. Retrieved 2007-10-18. Available at https://web.archive.org/web/20071028072110/http://info.tuwien. ac.at/iflt/safety/section1/1_1_1.htm

12 Lin CC, Chang CF, Lai MY, Chen TW, Lee PC and Yang WC: Far-infrared therapy: A novel treatment to improve access blood flow and unassisted patency of arteriovenous fistula in hemodialysis patients. J Am Soc Nephrol 18: 985-992, 2007. PMID: 17267744. DOI: 10.1681/ASN.2006050534

13 Yamashita K: The effects of the far-infrared ray (FIR) energy radiation on living body. In: Blood cell-an overview of studies in hematology. Moschandreou TE (eds.). Rijeka, Croatia, Europe: In Tech-Open Access Publisher; p. 271-302, 2012. DOI: $10.5772 / 36005$

14 Lin CC, Hsieh YY, Liu WC, Lee MS and Lung CC: Biological effects of far-infrared-ray hot compression on superoxide anions in human. AMM 52-54: 297-302, 2011. DOI: 10.4028/ www.scientific.net/AMM.52-54.297

15 Lin CC, Chiang YS and Lung CC: Effect of infrared-C radiation on skin temperature, electrodermal conductance and pain of the upper limbs in hemiparetic stroke patients. Int J Rad Biol 91(1): 42-53, 2014. DOI: 10.3109/09553002.2014.937512

$16 \mathrm{Su} \mathrm{LH}, \mathrm{Wu}$ KD, Lee LS, Wang H and Liu CF: Effects of far infrared acupoint stimulation on autonomic activity and quality of life in hemodialysis patients. Am J Chin Med 37(2): 215-226, 2009. PMID: 19507267. DOI: 10.1142/S0192415X09006783

17 Dover JS, Phillips TJ and Arndt KA: Cutaneous effects and therapeutic uses of heat with emphasis on infrared radiation. J Am Acad Dermatol 20(2 Pt 1): 278-286, 1989. PMID: 2644317.

18 Chen Y, Chen JC and Tseng SH: Tetrandrine suppresses tumor growth and angiogenesis of gliomas in rats. Int J Cancer 124: 2260-2269, 2009. PMID: 19165864. DOI: 10.1002/ijc.24208

19 Pistollato F, Abbadi S, Rampazzo E, Persano L, Della Puppa A, Frasson C, Sarto E, Scienza R, D'avella D and Basso G: Intratumoral hypoxic gradient drives stem cells distribution and MGMT expression in glioblastoma. Stem Cells 28(5): 851-862, 2010. PMID: 20309962. DOI: 10.1002/stem.415

20 Treps L, Perret R, Edmond S, Ricard D and Gavard J: Glioblastoma stem-like cells secrete the pro-angiogenic VEGF-A factor in extracellular vesicles. J Extracell Vesicles 6(1): 1359479, 2017. PMID: 28815003. DOI: 10.1080/20013 078.2017.1359479

21 Sonoda Y, Kanamori M, Deen DF, Cheng SY, Berger MS and Pieper RO: Overexpression of vascular endothelial growth factor isoforms drives oxygenation and growth but not progression to glioblastoma multiforme in a human model of gliomagenesis. Cancer Res 63(8): 1962-1968, 2003. PMID: 12702589.

22 Kil WJ, Tofilon PJ and Camphausen K: Post-radiation increase in VEGF enhances glioma cell motility in vitro. Radiat Oncol 7: 25, 2012. PMID: 22356893. DOI: 10.1186/1748-717X-7-25

$23 \mathrm{Lu} \mathrm{W}$, Melancon MP, Xiong C, Huang Q, Elliott A, Song S, Zhang R, Flores LG 2nd, Gelovani JG, Wang LV, Ku G, Stafford $\mathrm{RJ}$ and Li C: Effects of photoacoustic imaging and photothermal ablation therapy mediated by targeted hollow gold nanospheres in an orthotopic mouse xenograft model of glioma. Cancer Res 71(19): 6116-6121, 2011. PMID: 21856744. DOI: 10.1158/00085472.CAN-10-4557

24 You J, Shao R, Wei X, Gupta S and Li C: Near-infrared light triggers release of Paclitaxel from biodegradable microspheres: photothermal effect and enhanced antitumor activity. Small 6(9): 1022-1031, 2010. PMID: 20394071. DOI: 10.1002/smll.201 000028

25 Agarwal A, Mackey MA, El-Sayed MA and Bellamkonda RV: Remote triggered release of doxorubicin in tumors by synergistic application of thermosensitive liposomes and gold nanorods. ACS Nano 5(6): 4919-4926, 2011. PMID: 21591812. DOI: $10.1021 / \mathrm{nn} 201010 \mathrm{q}$

26 Day ES, Thompson PA, Zhang L, Lewinski NA, Ahmed N, Drezek RA, Blaney SM and West JL: Nanoshell-mediated photothermal therapy improves survival in a murine glioma model. J Neurooncol 104(1): 55-63, 2011. PMID: 21110217. DOI: $10.1007 / \mathrm{s} 11060-010-0470-8$ 
27 Inoué S and Kabaya M: Biological activities caused by farinfrared radiation. Int J Biometeorol 33(3): 145-150, 1989. PMID: 2689357.

28 Yu SY, Chiu JH, Yang SD, Hsu YC, Lui WY and Wu CW: Biological effect of far-infrared therapy on increasing skin microcirculation in rats. Photodermatol Photoimmunol Photomed 22: 78-86, 2006. PMID: 16606412. DOI: 10.1111/ j.1600-0781.2006.00208.x

29 Ishibashi J, Yamashita K, Ishikawa T, Hosokawa H, Sumida K, Nagayama $M$ and Kitamura $S$ : The effects inhibiting the proliferation of cancer cells by far-infrared radiation (FIR) are controlled by the basal expression level of heat shock protein (HSP) 70A. Med Oncol 25: 229-237, 2008. PMID: 17968683. DOI: $10.1007 / \mathrm{s} 12032-007-9020-4$
30 Bidwell GL 3rd, Perkins E, Hughes J, Khan M, James JR and Raucher D: Thermally targeted delivery of a c-Myc inhibitory polypeptide inhibits tumor progression and extends survival in a rat glioma model. PLoS One 8(1): e55104, 2013. PMID: 23372821. DOI: 10.1371/journal.pone.0055104

Received April 9, 2019

Revised June 10, 2019

Accepted June 18, 2019 